\title{
Enfance
}

http://www.necplus.eu/ENF

Additional services for Enfance:

Email alerts: $\underline{\text { Click here }}$

Subscriptions: Click here

Commercial reprints: $\underline{\text { Click here }}$

Terms of use : $\underline{\text { Click here }}$

\section{L'autisme raconté aux enfants : la littérature de jeunesse, un support de sensibilisation pertinent ?}

Lise Lemoine, Marie-Claude Mietkiewicz et Benoît Schneider

Enfance / Volume 2016 / Issue 02 / June 2016, pp 231 - 245

DOI: 10.4074/S0013754516002056, Published online: 06 July 2016

Link to this article: http://www.necplus.eu/abstract_S0013754516002056

How to cite this article:

Lise Lemoine, Marie-Claude Mietkiewicz et Benoît Schneider (2016). L'autisme raconté aux enfants : la littérature de jeunesse, un support de sensibilisation pertinent?.

Enfance, 2016, pp 231-245 doi:10.4074/S0013754516002056

Request Permissions : $\underline{\text { Click here }}$ 


\title{
L'autisme raconté aux enfants : la littérature de jeunesse, un support de sensibilisation pertinent?
}

\author{
Lise Lemoine, Marie-Claude MietKIEwiCZ \\ et Benoit SCHNEIDER*
}

\section{RÉSUMÉ}

Les ouvrages de jeunesse sur le thème du handicap peuvent être un support pour accompagner les situations éducatives ou pédagogiques. Cet article propose une analyse d'un corpus de 22 ouvrages francophones pour enfants de 3 à 11 ans, publiés depuis 2000, abordant la question de l'autisme. Nous essayons de dégager la représentation qu'ils proposent des enfants avec autisme en nous attachant plus particulièrement à leurs comportements. L'analyse thématique permet de dégager six caractéristiques des enfants avec autisme qui apparaissent dans au moins la moitié des livres : 1 . des enfants qui ont des comportements étranges et dérangeants, 2 . des enfants qui ne nous voient pas et ne nous entendent pas, 3 . des enfants qui vivent dans un autre monde, 4 . des enfants qui ne jouent pas et ont des rituels, 5 . des enfants qui ne communiquent pas, 6 . des enfants qui présentent une hypersensibilité. Nous croisons ensuite cette description avec les données scientifiques actuelles en nous appuyant sur les critères diagnostiques du DSM-5. On observe que la littérature jeunesse offre un panorama exhaustif et bien documenté des troubles, constat que nous tentons de compléter et d'expliquer par plusieurs spécificités éditoriales, mais qui autorise quelques premières réflexions quant aux potentialités éducatives de cette littérature.

MotS-CLEFS : HANDICAP, AUTISME, LITTéRATURE DE JEUNESSE

*Laboratoire INTERPSY (EA 4432), Université de Lorraine, Campus Lettres et Sciences Humaines, 23 Bd Albert 1er, 54015 Nancy Cedex, France. Emails : lise.lemoine@univ-lorraine.fr, marie-claude.mietkiewicz@univ-lorraine.fr benoit.schneider@univ-lorraine.fr 


\begin{abstract}
Autism told to Children:

Can Children's Literature be Used to Promote Awareness?

Children's books about disability can be used with an educational purpose. This paper offers the analysis of a corpus comprised of 22 books written in French for children from 3 to 11 years old. Published 2000 and later, they deal with the issue of autism. We describe the kind of representation of autism spectrum disorder that the books propose, particularly as regards the children's behavior. Thematic analysis reveals six characteristics that appear in at least half of the books: 1 . children with strange and upsetting behaviors; 2. children who cannot see us or hear us; 3. children who live in another world; 4. children who do not play or who have rituals; 5 . children who do not communicate; 6 . children who are hypersensitive. We then compare this description with current scientific data, based on DSM-5 diagnostic criteria. We show that children's literature offer a complete and well-documented account of the disorder. This can be explained by a number of editorial specificities such as promotion of knowledge concerning handicap or family involvement. It also offers views about how such books can be used in an educational context.
\end{abstract}

KEY-WORDS: DISABILITY, AUTISM, CHILDREN'S LITERATURE 


\section{INTRODUCTION}

À la fois miroir parce qu'elle permet aux enfants de se reconnaître dans les personnages et l'histoire racontée, et filtre parce qu'elle autorise prise de distance et positionnement face aux situations évoquées (Léon, 2004 ; Chartier, 2010), la littérature jeunesse remplit des fonctions de récréation, d'édification et d'éducation (Chelebourg \& Marcoin, 2007). Quand il aborde des sujets difficiles, le récit fictionnel peut se révéler d'un grand intérêt pour aborder certaines problématiques développementales ou éducatives (Schneider \& Mietkiewicz, 2013) ; ainsi, les ouvrages sur le thème du handicap, apparus dès le XIX ${ }^{\mathrm{e}}$ siècle ont fait émerger un nouvel usage pédagogique des livres pour enfants en ce qu'ils peuvent être un support pour engager le dialogue sur la différence, questionner les réactions spontanées et susciter la réflexion (Joselin, 2008, 2013 ; Lemoine, Mietkiewicz, \& Schneider, 2012).

Parmi les ouvrages abordant la thématique du handicap, nous nous intéresserons à ceux qui abordent la question de l'autisme afin de dégager la représentation qu'ils proposent de l'enfant avec autisme et de montrer dans quelle mesure ils peuvent contribuer à sensibiliser le jeune lecteur à sa singularité, l'accompagner lorsque cet enfant fait partie par exemple de son univers familial, le préparer à le rencontrer dans différents contextes de socialisation avec moins d'appréhension lorsque les circonstances l'y amènent.

$\mathrm{Si}$ la question de la représentation sociale de l'autisme a été abordée de longue date, elle est restée d'abord circonscrite à des réseaux spécialisés (Courtail \& Gourdon, 1997). Les larges débats et les enjeux médico-psycho-sociaux qui ont marqué l'évolution des prises en charge ont eu des effets sur l'évolution des représentations sociales de l'autisme qui ont eu à leur tour " un effet rétroactif sur sa propre définition avec pour corollaire une évolution positive sur la stigmatisation des personnes atteintes et leurs parents »(Chamak, 2013, cité in Guinchat, p.12). L'étude de ces représentations chez l'adulte a donné lieu à des travaux récents (Guelai, 2015 ; Hamhami, 2015), circonscrits à des publics spécifiques (chercheurs, associations, professionnels), mais n'a pas encore suscité de recherche chez l'enfant. Notre approche représente une voie pour y contribuer et ce par une double approche : elle interroge les représentations des concepteurs d'ouvrages (auteurs, éditeurs. . .) ainsi que celles des publics destinataires (enfants lecteurs, parents, éducateurs) et peut au final contribuer à l'analyse des « enjeux sous-jacents à la mise en place des représentations qui s'élaborent, se modifient, se confrontent, (qui) constitue une étape essentielle pour comprendre comment les déterminants sociaux et culturels interviennent dans la construction d'une maladie ». (Chamak, 2010, p 150).

Après avoir explicité les critères d'inclusion des ouvrages de jeunesse dans le corpus d'étude, nous dégagerons les caractéristiques majeures des enfants avec autisme qui y sont explicitement décrits comme tels. Ensuite, nous confronterons ces présentations aux critères scientifiques qui conduisent au diagnostic de ce trouble. Il ne s'agit bien évidemment pas d'expertiser des ouvrages de jeunesse 
qui n'ont pas vocation à être des " manuels pour enfants » au regard de leur adéquation avec des connaissances scientifiques. Cependant cette mise en regard nous semble intéressante afin d'apprécier la pertinence des supports mis à disposition des enfants et de conclure sur l'intérêt et l'usage éventuel de ces livres dans une perspective de sensibilisation de jeunes lecteurs susceptibles de côtoyer dans leur entourage familial, environnement scolaire ou amical, des enfants avec autisme ou d'accompagnement dans des structures de prise en charge spécialisées tels les Centres Ressources Autisme.

\section{MÉthodologie}

\section{Constitution du corpus}

Parmi les nombreux ouvrages à destination des jeunes lecteurs qui approchent la problématique du handicap, nous avons cherché à faire un inventaire exhaustif des livres publiés depuis le début du $\mathrm{XXI}^{\mathrm{e}}$ siècle qui abordent la question de l'autisme. En croisant les résultats de nos recherches sur les sites dédiés à la littérature jeunesse, les informations fournies par des associations ou le réseau Canopé ${ }^{1}$ et les conseils dispensés par bibliothécaires et libraires, nous avons constitué une première liste de titres sur laquelle nous avons, afin de garantir une certaine homogénéité du corpus, procédé à une sélection sur la base de critères précis d'âge du lectorat, de forme et de contenu.

Nous avons retenu les livres à destination d'enfants dont les âges s'échelonnent de 3 à 11 ans et habituellement scolarisés dans les écoles maternelles et élémentaires. Nous avons inclus les seuls livres qui s'inscrivent dans la catégorie des récits fictionnels où l'enfant-lecteur est invité à suivre la trame narrative d'une histoire dont un des protagonistes est un enfant avec autisme. Nous n'avons pas retenu ici des ouvrages destinés aux enfants qui s'apparentent à des « manuels » de sensibilisation. Nous en avons mentionné pour information quelques-uns en complément de l'Annexe 1 ( $c f$. Corpus des ouvrages de jeunesse). Enfin, à l'issue d'une lecture attentive des ouvrages qui remplissent ces deux premières conditions, nous avons retenu ceux dans lesquels le récit concerne un personnage atteint de troubles présentés sans aucune ambiguité comme relevant de l'autisme ; la présence explicite de cette information nous semble indispensable à la rigueur de notre approche afin de ne prendre en considération que les livres dans lesquels le jeune lecteur est informé du diagnostic porté et peut ainsi rapporter les particularités de cet enfant au trouble dont il est atteint.

Les 22 livres qui constituent notre corpus (cf. Annexe 1) sont donc exclusivement des livres dans lesquels l'autisme d'un des personnages est porté à la connaissance du lecteur, parfois dès le titre $[4,10,12,13,15,22]$, le plus souvent au tout début du récit.

1 Le réseau Canopé, placé sous la tutelle du ministère de l'Éducation nationale, de l'enseignement supérieur et de la recherche, édite des ressources pédagogiques transmédia répondant aux besoins de la communauté éducative (https://www.reseau-canope.fr/qui-sommes-nous.html) 
Parus depuis 2000 à un rythme constant de 7 ou 8 livres par tranche de 5 années, la plupart des ouvrages ont été publiés en français pour leur édition originale, 13 en France et 7 au Québec, 1 est traduit de l'américain et 1 autre du coréen. 7 de ces livres s'adressent à des enfants de $3-5$ ans $[1,12,13,15,18$, $19,22], 11$ à des enfants de $6-8$ ans $[2,3,4,6,9,10,11,16,17,20,21]$ et 4 à des enfants de 9-11ans [5, 4, 10, 14].

\section{Analyse de contenu des ouvrages}

Pour rendre compte de la richesse du matériel tout en adoptant une méthode la plus objective possible, nous avons procédé à une analyse de contenu thématique après construction d'une grille permettant de repérer les caractéristiques essentielles des héros avec autisme, et les particularités de leurs comportements. Pour ce faire nous nous sommes référés pour la présente analyse aux critères diagnostiques tels que proposés par la CIM 10 (OMS CIM-10, 1999). Nous avons tenté de repérer dans chacun des ouvrages, par une procédure d'analyse de contenu thématique qualitative, chacune des unités textuelles (expression, phrase, élément de dialogue) qui nous est apparu «illustrer» les critères diagnostiques de l'autisme.

\section{RÉSULTATS ET ANALYSE}

Les personnages avec autisme des livres sont très majoritairement de sexe masculin $(17 / 22)$, ils sont habituellement désignés par leur prénom, rarement par des vocables en rapport avec leurs singularités (Paul-la-Toupie, princesse Libellule, TED). À la différence d'autres enfants porteurs de handicap(s) ou de pathologie(s) (Joselin, 2008, 2013), ils ne présentent aucune particularité physique significative et les seules mentions relatives à leur apparence renvoient à leur beauté remarquable $[6,8,14]$.

La différence est abordée comme une maladie dans 6 livres [5, 11, 14, 16, 20, 22] comme une déficience dans 6 autres $[2,5,14,15,20,21]$ avec une insistance sur l'écart entre l'âge développemental et l'âge chronologique (" copain était plus grand que nous, mais il était encore bébé dans sa tête » [2] ; "elle a huit ans et [...] elle agit comme une enfant de deux ans » [14]). En revanche, on trouve dans 7 livres $[4,7,10,12,14,16,20]$ mention de capacités particulières, notamment mnésiques hors du commun («un magnétophone ne ferait pas mieux » [16]), pour retenir, par exemple, le plan du métro [7], les fréquences radio [10] ou les dates [14].

L'analyse thématique permet de dégager six caractéristiques des enfants avec autisme qui apparaissent dans au moins la moitié des 22 livres ; nous les présenterons par ordre de fréquence d'apparition en illustrant chacune par quelques exemples. 
Six caractéristiques essentielles

\section{Des enfants qui ont des comportements étranges et dérangeants (21 livres)}

La caractéristique la plus souvent mentionnée concerne des comportements qualifiés de bizarres, d'étranges, de spéciaux, voire de mystérieux.

En premier lieu, 16 livres $[2,5,6,7,8,10,11,12,13,14,17,18,19,20$, $21,22]$ évoquent des manières singulières de se mouvoir dans l'espace et de se déplacer ( en dansant » 6,12$]$, « sur la pointe des pieds » $[8,12,13]$, « sur le bout des orteils » [20], « pieds nus » [13], « à petits pas rapides, saccadés » [14], " précipités » [6], « comme une petite souris » [13] ou " comme un boiteux » [21]), auxquelles s'ajoutent souvent des gestes amples et répétitifs des mains $[2,8,12$, $13,14,22]$ qui peuvent évoquer un essuie-glace [2] ou un moulin à vent [12]. Des mouvements de rotation rapide $[6,7,8,13]$, « ronde étourdissante » [6] ou «toupie » [7] et de balancement [5, 19, 22] sont également rapportés.

Les cris ou hurlements sont présents dans 10 livres $[1,2,5,6,8,9,10,11$, $12,19]$. Les récits mettent l'accent sur la puissance des émissions vocales : «Petit soleil crie toujours très fort » [1] ; «il hurle si fort qu'on l'entend même dehors » [12] et sur leur démesure : Timothée " fait la sirène du camion des pompiers » parce que « les frites mettent trop de temps à cuire » [19]. Les colères sont bruyantes, incontrôlables et si dévastatrices et effrayantes [2, 6, 14, 21] qu'elles sont comparées à des catastrophes météorologiques : tempête [5, 7], tornade [2], tourbillon [8], ouragan [12], elles font trembler les murs [9], s'accompagnent de gestes agressifs $[1,2,5,10,12]$ et d'automutilation $[5,17,18,21]$. Il arrive aussi que ces enfants rient aux éclats, mais c'est alors «sans qu'on sache pourquoi » [6] ou « pour rien » [20], voire en décalage absolu avec le ressenti : « il se sent mal et ne sait pas comment l'exprimer, alors il rit, il s'agite et se balance d'avant en arrière » [22] ou avec les circonstances : « il lui arrive même de rire alors que je suis fâché » [10].

D'autres comportements dérangent parce qu'ils vont à l'encontre des règles de la vie en collectivité $[10,13,14,16,19]$ : " tous les enfants du groupe viennent s'asseoir pour manger. Éloi, lui, continue à jouer avec les autos » [13] ou ne respectent pas les conventions sociales [10, 19]. Mentionnons aussi la comparaison fréquente (15 livres) avec un animal sauvage [2, 8, 9] ou familier (libellule [3], chauve-souris [8], chat [8], chien fou [8], petite souris [13], mouette [17]...) pour décrire les conduites des enfants avec autisme.

\section{Des enfants qui ne nous voient pas et ne nous entendent pas (18 livres)}

La seconde particularité, soulignée dans 18 livres $[1,2,3,4,5,7,8,9,10,11,12$, $13,14,16,17,18,20,21]$ vise des enfants qui ne semblent ni voir ni entendre les personnes qui tentent de capter leur attention : «c'est comme si elle était aveugle et sourde, et pourtant, elle voit et elle entend ", " elle fixait toujours un point imaginaire, la tête penchée de côté », " elle regarde à travers les gens comme s'ils n'étaient pas là » [14]. Ils ignorent le plus souvent les sollicitations verbales ainsi, «Paul ne répond jamais » [11], « on dirait qu’Éloi ne m’entend pas » [13]. 
Alors qu'ils ne réagissent pas aux stimulations de leur entourage, ces enfants sont parfois fascinés par des choses insolites ou sans intérêt qu'ils observent longuement : le petit nez d'un lapin qui remue sans cesse [2], l'eau qui coule dans le siphon du lavabo [10] ou tout ce qui est rond : la lune, les panneaux de signalisation, la pizza, les roues et les bouches d'égout [17].

\section{Des enfants qui vivent dans un autre monde (15 livres)}

Les enfants avec autisme sont présentés comme étrangers à leur environnement. Ils vivent ailleurs $[1,3,9,14,16,17,18,21]$, sur une planète inconnue $[2,22]$, dans la lune [7,17,20], sur la branche d'une étoile [3], dans les nuages [16], à la recherche du soleil [13]. Extraterrestres, martien, cosmique [8] voire Alien [5], ils sont solitaires et inaccessibles, prisonniers d'une bulle $[3,4]$ dont « on ne sait pas s'il existe une clef, une formule magique, un code secret. . pour y accéder» [3], enfermés dans une forteresse [5], « dans un cocon enroulé dans les rayons lunaires » [7]. Leur monde est aussi en eux $[8,9]$ : « en fait, ils vivent seuls audedans d'eux dans une autre dimension qui nous est inconnue » [8].

\section{Des enfants qui ne jouent pas et ont des rituels (14 livres)}

Ces enfants ne jouent pas comme les autres ni avec les autres $[1,2,5,7,9,10$, $11,13,14,16,17,18,20]$ : " c'est juste qu'il ne SAIT pas jouer » ${ }^{2}$ [1] mais ils s'absorbent dans des activités ritualisées et solitaires $[1,4,7,8,9,10,11,12$, $13,16,17,18,19,21]$ : faire tourner les roues d'une petite voiture [1], aligner les personnages de Walt Disney [8] ou ramasser les branches mortes aux pieds des arbres [21]. Ils établissent des collections diverses (boules de papier froissé [7], poussières [9], couvercles de yaourts [19]), trient, ordonnent, alignent les éléments et ne supportent pas le moindre dérangement dans l'ordonnancement des choses : «chaque avion doit rester exactement à sa place » [4]. Non seulement leur activité est stéréotypée $[1,4,7,8,9,10,11,17]$, mais ils aiment la pratiquer au même endroit [7, 9, 17], emprunter les mêmes itinéraires [17], regarder les mêmes émissions ou vidéos $[8,10,19]$, écouter les mêmes musiques [8] et redoutent toute nouveauté.

\section{Des enfants qui ne communiquent pas (12 livres)}

Les enfants avec autisme ne parlent pas $[2,5,6,8,9,14,17,20]$ : « elle n'essaye jamais de nous dire quelque chose. Elle ne communique pas du tout» [14]. Et s'ils produisent des émissions verbales, c'est sans rechercher l'échange si bien qu'il n'est pas possible d'entretenir une conversation avec eux [4, 5, 7] soit qu'ils produisent inlassablement les mêmes mots ou les mêmes bruits $[7,16,20]:$ « mon petit frère aime faire des sons avec sa bouche, toujours les mêmes sons. De plus en plus fort: Woa woa! Taou taou ! Trrrrrout trrrrrout !» [20], soit qu'ils répètent en écho ce qu'on dit [10, 18, 22]. 


\section{Des enfants qui présentent une hypersensibilité (12 livres)}

Alors qu'ils semblent indifférents au monde qui les entoure, ces enfants sur-réagissent à certains bruits ordinaires et anodins ou inaudibles pour tout un chacun $[2,3,4,5,13]$ comme " un coup de klaxon, une porte qui claque, une feuille qui tombe d'un arbre » ou « le bruit du silence » [5], « le Cocorico d'un coq» [16], le ronronnement de l'aspirateur [17].

Cette hypersensibilité concerne aussi les odeurs [4, 16], les saveurs [13, 18, 20] et le toucher ; en effet, ils ne supportent pas d'être portés [17], câlinés [13] de donner la main [14] ou refusent le moindre contact physique $[2,4,14,18]$.

\section{Les correspondances avec les critères diagnostiques du trouble du spectre de l'autisme}

Les 22 livres sur lesquels porte notre analyse n'ont pas vocation à refléter les critères diagnostiques de l'autisme ; en effet, ils s'adressent aux jeunes enfants et ne peuvent que prétendre leur apporter des informations susceptibles de les aider à approcher l'enfant avec autisme en connaissance des particularités de ses conduites et attitudes, c'est pourquoi nous avons choisi de faire apparaître et catégoriser les comportements les plus fréquemment mentionnés à partir du point de vue de l'enfant partenaire du sujet avec autisme.

Il est cependant intéressant de reprendre cette analyse en regard des données scientifiques afin de déterminer si ces ouvrages peuvent éclairer les jeunes lecteurs sur ce qui fait consensus pour définir l'autisme. Jusqu'à une date récente la notion de «triade autistique » était largement partagée (OMS CIM-10 : 10 e révision, 1999, DSM IV, 2003), mais vient d'être récemment publié le DSM-5 (2015) ; la publication de CIM-11, annoncée pour 2015, ne nous a pas été accessible dans sa version complète au moment de la rédaction de cet article (Pull, 2014), mais les experts des deux révisions travaillent en concertation tout en gardant leur autonomie de rédaction. L'approche catégorielle adoptée dans le DSM IV a été réinfléchie dans le DSM-5 vers une approche davantage dimensionnelle de la sévérité de l'autisme. La définition de l'autisme du DSM-IV était donc caractérisée par 3 symptômes de base (triade autistique) ; dans le DSM-5 deux catégories de symptômes subsistent $(\mathrm{A})$ : «troubles de la réciprocité sociale » et «troubles du langage/de la communication » ont été regroupés en " déficits persistants de la communication sociale observés dans des contextes variés »; (B) « caractère restreint et répétitif des comportements, des intérêts ou des activités ». C'est en référence à des caractéristiques génériques que nous examinerons les liens entre description scientifique et contenus des ouvrages. Des exemples tirés des ouvrages sont présentés en Annexe 2 afin d'illustrer chacune de ces correspondances.

Même si les livres mettent l'accent sur certaines caractéristiques des enfants avec autisme parce qu'elles sont particulièrement saillantes et dérangeantes pour leurs pairs, on constate que la littérature jeunesse offre un panorama exhaustif et bien documenté des symptômes. En effet, ce qui pour l'enfant-lecteur relève 
des comportements étranges et dérangeants renvoie à la fois au « déficit de la réciprocité sociale et émotionnelle », et au "déficit du développement, du maintien et de la compréhension des relations » (critère $\mathrm{A}$ ) et au " caractère restreint, répétitif et stéréotypé des comportements, des intérêts ou des activités » (critère B). La seconde caractéristique que nous avons dégagée Des enfants qui ne nous voient pas et ne nous entendent pas illustre, du point de vue du partenaire de l'enfant avec autisme confronté à la frustration d'une communication empêchée, renvoie aux «déficits des comportements de communication non verbaux utilisés au cours des interactions sociales" (critère A). Des enfants qui vivent dans un autre monde, qui ne jouent pas et ont des rituels, qui ne communiquent pas, qui ont une bypersensibilité, correspondent aux « déficits persistants de la communication et des interactions sociales » (critère $\mathrm{A}$ ) et du "caractère restreint, répétitif et stéréotypé des comportements, des intérêts ou des activités »(critère B). Au-delà de ces caractéristiques fondamentales, certaines des manifestations considérées comme non spécifiques - perturbations du sommeil et des conduites alimentaires, crises de colères et gestes auto agressifs - sont très présentes dans les livres où elles contribuent à illustrer les composantes déroutantes du comportement de l'enfant avec autisme.

\section{DISCUSSION ET CONCLUSION}

Comment ces livres destinés à des enfants susceptibles d'être confrontés dans leurs familles, dans des activités de loisirs ou à l'école à des enfants avec autisme peuvent-ils contribuer à aider les enfants typiques à décrypter les conduites des enfants avec autisme et à les fréquenter le plus sereinement possible, c'est-à-dire à la fois sans les redouter et sans les heurter?

Un premier niveau de réponse concerne, comme on l'a vu, la richesse et l'étendue de la description des comportements. Un second point important concerne le public visé ; les questions soulevées sont à examiner en regard des âges des destinataires, des enfants de 3 à 11 ans, axe de lecture que l'espace de cet article ne nous a pas permis de développer. Pour les tout-petits la question est abordée à travers l'histoire d'un petit chiot [16], pour les grands (9-11) l'approche est plus directe, plus crue : «calvaire pour la famille » [5], ou plus documentée : " recherche de la définition dans le dictionnaire » ou référence à l'étymologie et aux travaux de Bettelheim [8]. Deux composantes importantes complémentaires caractérisent ces ouvrages et viennent soutenir et « colorer» leur orientation.

\section{Des ouvrages à vocation et à structuration pédagogiques}

Presque tous les ouvrages (17) incluent des explications (jusqu'à une dizaine de pages) sur l'autisme ("C'est quoi être autiste ? Ça touche qui ? Est-ce qu'on peut guérir de l'autisme ?» [1]). La majorité des informations portent sur les caractéristiques des enfants (leurs difficultés relationnelles, de communication, dans l'expression des émotions et sentiments, leur isolement dans leur monde, leurs rituels et stéréotypies...) ; elles peuvent aussi porter sur l'épidémiologie, l'étiologie, les programmes de prise en charge ou la législation. Cela peut prendre 
la forme de «notes aux parents » ou « au lecteur », d'une invitation à « découvrir ensemble » pour " parler autour de l'album », " de fiches pédagogiques », de " questions à travailler avec l'enfant», d'un supplément de jeux ou d'un support DVD, pour exemple : «Pour mieux comprendre cette différence, vous trouverez dans ce livre des renseignements utiles ainsi qu'une référence internet afin d'en connaitre davantage sur le sujet ou d'obtenir du soutien. De plus, pour vous permettre d'exploiter et d'approfondir le sujet traité, des questions et une idée d'activité vous sont proposées » [12]. Ces ouvrages se veulent « témoignage sur la différence » [21] et guide pour l'accompagnement d'un enfant avec autisme qui : " provoque nécessairement des questions, des réactions et des inquiétudes » [22] ; nombre d'entre eux s'adressent délibérément au pédagogue en même temps qu'à l'enfant : "Cet ouvrage [...] a pour objectif de rendre accessible à l'enfant qui lit, un handicap complexe. [...] Il donne à l'enfant des éléments d'information visant à le sensibiliser et à l'ouvrir à la différence » [10].

\section{Des ouvrages « militants » et des auteurs concernés}

Treize livres apparaissent clairement militants : trois [12, 13, 16] sont publiés dans des collections dont l'objectif est de favoriser l'intégration des enfants avec handicap ( La collection [...] a pour but d'aider les familles et les parents d'enfants porteurs d'un handicap à mieux communiquer autour de leur pathologie et à faciliter leur intégration scolaire et extrascolaire » [16]). Quatre $[7,15,19,20]$ mettent en exergue les difficultés auxquelles les familles sont confrontées ("C'est difficile d'avoir un enfant différent. C'est comme un enfant d'une autre planète dont on n'aurait pas le mode d'emploi » [7]), cinq autres [3, 9, 11, 18, 22] invitent à la tolérance ("La "différence" est tangible, offrons donc aux enfants les outils pour se comprendre un peu mieux !» [22]). En outre, pour quatre $[10,12,16,22]$ de ces livres il est annoncé qu'une partie des droits d'auteurs sera reversée à des associations en faveur des personnes avec autisme.

La moitié des ouvrages sont écrits par des auteurs concernés par l'autisme. Les auteurs de 6 livres $[7,8,9,17,20,22]$ précisent qu'ils sont mère, père ou grand-mère d'un enfant avec autisme ; un livre est écrit par les membres d'une association en faveur des personnes avec autisme [10], les auteurs de 4 autres récits, enseignante, psychoéducatrice, orthophoniste. [13, 16, 18, 19] ont rencontré des enfants avec autisme dans le cadre de leur activité professionnelle. Un auteur dédie le livre «à tous les enfants Soleil qui m’ont inspirée et me donnent tant de plaisir à travailler avec eux » [1], un autre raconte, qu'enfant, il « s'est lié d'amitié avec un enfant autiste » [21].

\section{Vers un regard d'analyse critique plus étendu}

Livres témoignages, livres militants et pédagogiques... Notre choix d'analyse pour le présent article de mettre l'accent sur les données comportementales/diagnostiques majore sans doute la dimension "documentée » des situations. Cette approche n'épuise bien évidemment pas toutes les potentialités des ouvrages, ni du point de vue de leurs ressources, ni du point de vue de 
l'analyse critique. Nous y avons fait allusion avec Joselin $(2008,2013)$ : nombre de thématiques méritent intérêt en ce qu'elles peuvent permettre à enfants et éducateurs d'aborder les contextes de vie (fratrie, scolarisation...) d'enfants avec troubles neuro-développementaux, et aux seconds d'aider les premiers à décrypter les conduites des enfants avec autisme, à aborder les émotions qu'ils éprouvent et à ajuster leurs échanges le plus sereinement possible ; elles offrent une voie d'entrée originale pour examiner les rapports entre évolution des connaissances et représentations sociales de l'autisme.

\section{RÉFÉRENCES}

Chamak, B. (2010). Autismes : des représentations multiples, sources de controverses, Enfances \& Psy, 2(47), 150-158.

Chamak, B. (2013). Le militantisme des associations d'usagers et de familles : l'exemple l'autisme. Sud/Nord, 25, 71-80.

Chamak, B. \& Cohen, D. (2007). Transformations des représentations de l'autisme et de sa prise en charge, Perspectives Psy, 3(46), 84

Chartier, A. M. (2010). La lecture des jeunes et les institutions éducatives : de la transmission à la médiation, Forumlecture.ch [Online], 4, p. 1-18. Page consultée le 28 août 2014. http://www.forumlecture.ch

Chelebourg, C. \& Marcoin, F. (2007). La littérature de jeunesse. Paris : Armand Colin.

Courtial, J. P. \& Gourdon, L. (1997). Autisme et représentations sociales des chercheurs : Une approche historique des recherches contemporaines sur l'autisme. Neuropsychiatrie de l'enfance et de l'adolescence, 45(10), 573-583.

Crocq, M.-A. \& Guelfi, J.-D. (Coord.) (2015). DSM-5. Manuel diagnostique et statistique des troubles mentaux. American Psychiatric Association. Issy-les-Moulineaux : Elsevier Masson.

Guelai, A. T. (2015). Critères de différenciation dans les représentations sociales des enfants autistes chez les éducateurs. Thèse de doctorat Psychologie, Université Paul Valérie Montpellier, $131 \mathrm{p}$.

Guinchat, V. (2014). Les comorbidités cliniques de l'autisme : une interface entre le syndrome autistique et ses causes. Neurosciences [q-bio.NC]. Université Pierre et Marie Curie Paris VI, 2014. Français. <NNT : 2014PA066366>. < tel-01139236=

Hamhami, M. A. (2015). Représentations sociales et catégorisation du handicap. Le cas de l'autisme. Thèse de doctorat de psychologie, Tlemcen (Algérie) : Université d'Abou Bekr Belkaid, 175 p.

OMS. CIM 10. (1999). Classification statistique international des maladies et des problèmes de santé connexes. $10^{\mathrm{e}}$ Révision. Genève : OMS.

Joselin, L. (2008). Les représentations du handicap dans la littérature jeunesse française et italienne. Unpublished doctoral dissertation. Université de Rouen.

Joselin, L. (2013). Représentations plurielles des héros en situation de handicap dans la littérature jeunesse. In Schneider, B., \& Mietkiewicz, M.-C. (Dir.), L'enfant dans les livres. Représentations, savoirs, normes (pp. 103-115). Toulouse : Érès. 
Lemoine, L., Mietkiewicz, M.-C., \& Schneider, B. (2012). L'enfant porteur de trisomie 21 et sa fratrie dans la littérature jeunesse. Revue Francophone de la Déficience Intellectuelle, 23, 43-55.

Léon, R. (2004). La littérature de jeunesse à l'école : Pourquoi? Comment? Paris : Hachette livre.

Pull, C. B. (2014). DSM-5 et CIM-11, Annales Médico-Psychologiques, 172, 677-680.

Schneider, B. \& Mietkiewicz, M.-C. (2013). Les enfants dans les livres. Représentations, savoirs, normes. Toulouse : Érès.

\section{AnNexe 1 : Corpus des ouvrages de Jeunesse}

[1] Bagla, C. (2013). La voiture de petit soleil. Vichy : Éditions Aedis.

[2] Beaudoin, J. (2009). Mon copain Marc. Marbache : Oxalide « Kid's Corner».

[3] Distinguin-Rabot M. C. (2012). Le fil d'argent. Rezé : Alix Éditions.

[4] Doering-Trouville, A. (2011). Mon ami est autiste. Saint-Denis : Chenelière Éducation.

[5] Jaoui, S. (2010). Je veux changer de sæur. Paris : Casterman, coll. « Roman Junior ». [6] Joséphine, R. (2006). La clé des songes. Rouen : Gecko jeunesse, coll. «Les motssésames ».

[7] Kochka (2000). Brelin la lune. Montréal : Hurtubise, coll. « Plus ».

[8] Kochka (2002). L'enfant qui caressait les cheveux. Paris : Grasset-Jeunesse , coll. « Lampe de Poche Pré-Ados ».

[9] Kochka (2002). Le liseron de Jules. Paris : Belin, coll. «Les albums de Justine».

[10] Larchez, M. (2001). J'ai un copain autiste. Colmar : Les Éditions d'Alsace.

[11] Laurencin, G. (2004). Paul-la-toupie : bistoire d'un enfant différent. Monaco : Éditions du Rocher, coll. «Lo Païs d'Enfance ».

[12] Marleau, B. (2013). Lolo. L'autisme. Sainte-Thérèse : Boomrang, coll. « Au cœur des différences ».

[13] Martel, S. (2008). Le monde d'Éloi : une histoire sur. . . l'autisme. Saint-Lambert : Enfants Québec, coll. «J'apprends la vie».

[14] Matthews-Martin, A. (2006). Le club des Baby-Sitters : nos plus grands défis. Le défi de Kristy. Paris : Gallimard Jeunesse.

[15] Moore-Mallinos, J. (2008). Mon frère est-il comme Einstein? Vivre avec l'autisme. St. Lambert : Héritage Jeunesse, coll. « Parlons-en !».

[16] Pezet, F. \& Caugant, C. (2009). Ted: une patte ici, trois pattes là-bas. Paris : P'tits Totems, coll. «Ta santé m'intéresse».

[17] Philibert, F. (2011). Mon petit frère de la lune. Rezé : Éditions d'un monde à l'autre.

[18] Roque, J. (2000). Timothée un élève différent. Nouméa : Autoédition « Josiane Roque».

[19] Roque, J. (2005). Timothée un frère différent. Nouméa : Autoédition « Josiane Roque ».

[20] Roy, D. (2010). Mon petit frère super héros. Montréal : Fonfon, coll. « Histoires de vivre ». 
[21] Song, J.H. (2008). Pibi, mon étrange ami. Paris : Le Sorbier.

[22] Tardif, J. (2010). L'antenne cassée on l'autisme expliqué aux tout-petits. Saint-Hubert : Corne de Brume, coll. « Prendre le vent ».

\section{OUVRAGES ET DOCUMENTS DESTINÉS AUX ENFANTS MAIS NE RELEVANT PAS DIRECTEMENT DE RÉCITS FICTIONNELS:}

Chadu (1998). Iséo et le jeune autiste. Sésame autisme. Saint-Fons : Chadu.

Deslauriers, S. (2012). Laisse-moi t'expliquer l'autisme : album documentaire pour mieux comprendre et mieux vivre la différence. Saint-Léon: Midi Trente Éditions.

Grand, C. (2012). Toi qu'on dit autiste : Le syndrome d'Asperger expliqué aux enfants. Paris : L'Harmattan.

Laurent, L, \& Cellier, V. (2008). Epsilon un enfant extraordinaire : Qu'est-ce que l'autisme? Colmar : Jérôme Do Bentzinger.

Laurent, L, \& Cellier, V. (2011). Epsilon, un écolier extraordinaire : Qu'est-ce que l'autisme à l'école? Mouans-Sartoux : AFD - Autisme France Diffusion.

\section{ANNEXE 2 : LES CORRESPONDANCES AVEC LES CRITĖRES DIAGNOSTIQUES DU TROUBLE DU SPECTRE DE L'AUTISME}

(Chaque critère est illustré par quelques courts exemples extraits des ouvrages : chaque ouvrage est cité une fois)

\section{A. «Déficits persistants de la communication et des interactions sociales [...]»}

1. «Déficits de la réciprocité sociale ou émotionnelle [...]»

ex. " anomalies de l'approche sociale et d'une incapacité à la conversation bidirectionnelle normale ", "difficultés à partager les intérêts, les émotions et les affects ", "jusqu'à une incapacité d'initier des interactions sociales ou d'y répondre»

«On dirait qu'il vit dans un monde à part, qu'il est "dans sa bulle" » [4]

«Et soudain, elle les abandonne, là, au bord d'une histoire, sur une branche d'étoile, sans leur dire un mot» [3]

«C’est la première fois que Brelin exprime clairement une idée dans une phrase qui se tient » [7]

«[...] commencera à se parler à voix haute » [15] 
2. «Déficits des comportements de communication non verbaux utilisés au cours des interactions sociales »

ex. " anomalies du contact visuel et du langage du corps ", " déficits dans la compréhension et l'utilisation des gestes ", "jusqu'à une absence totale d'expressions faciales et de communication non verbale»

« Il avait du mal à regarder les gens dans les yeux » [2]

«Elle regardait en l'air avec ses grands yeux clairs un truc que personne ne voit » [5]

«Comme son antenne est brisée, il ne pense pas à demander ce qui se passe » [22]

3. «Déficit du développement, du maintien et de la compréhension des relations $[\ldots] »$

ex. « difficultés à ajuster ses comportements à des contextes sociaux », " difficultés à partager des jeux imaginatifs ou à se faire des amis », «jusqu'à l'absence d'intérêt pour les pairs »

«Alors il ne peut pas vous imiter et apprendre de vous si facilement» [1]

«Tous les enfants du groupe viennent s'asseoir pour manger. Éloi, lui, continue à jouer avec les autos » [13]

«Elle a essayé de retirer sa main de la mienne en poussant un gémissement étrange » [14]

\section{B. «Caractère restreint et répétitif des comportements, des intérêts ou des activités [...]»}

1. «Caractère stéréotypé ou répétitif des mouvements, de l'utilisation des objets ou du langage»

ex. "stéréotypies motrices simples, activités d'alignement des jouets ou de rotation des jouets, écholalie, phrases idiosyncrasiques »

«Les doigts crispés de la princesse aînée » [6]

« Il répète aussi souvent la même phrase. Sa maman dit que cela le rassure, surtout quand il ne sait pas ce qu'il va se passer » [10]

« Il s'est mis à marcher sur le bout des orteils » [20] 
2. «Intolérance au changement, adhésion inflexible à des routines ou à des modes comportementaux verbaux ou non verbaux ritualisés »

ex. « détresse extrême provoquée par des changements mineurs, difficultés à gérer les transitions, modes de pensées rigides, ritualisation des formules de salutation, nécessité de prendre le même chemin ou de manger les mêmes aliments tous les jours »

«Matthieu aligne ses personnages de Walt Disney » [8]

«Parfois, inlassablement, il coulait des pierres dans un seau » [9]

« Rien à faire, il met tout en rang, par terre » [12]

\section{3. "Intérêts extrêmement restreints et fixes, anormaux soit dans leur intensité, soit dans leur but»}

ex. « attachement à des objets insolites ou préoccupations à propos de ce type d'objets ", " intérêts excessivement circonscrits et persévérants »

«Il retient immédiatement et intégralement une histoire qu'on lui raconte pour la première fois à condition que ça l'intéresse : un magnétophone ne ferait pas mieux!»[16]

«Comme il adore regarder l'eau tourner en rond, nous n'avions plus d'eau chaude pour nous laver» [19]

«Moi, je le regarde beaucoup ce petit frère qui a toujours les yeux tournés vers le ciel. Même quand on fait les courses, même en voiture » [17]

4. "Hyper ou hyporéactivité aux stimulations sensorielles ou intérêt inhabituel pour les aspects sensoriels de l'environnement »

ex. " indifférence apparente à la douleur ou à la température ", " réactions négatives à des sons ou des textures spécifiques, actions de flairer ou de toucher excessivement les objets, fascination visuelle pour les lumières ou les mouvements »

«Va chercher ce qui reste de la voiture, la ramasse, la regarde, dessus, dessous, devant, derrière, partout. .. » [11]

«Timothée a une sensibilité différente de la nôtre. Un contact physique anodin peut quelquefois l'effrayer » [18]

« Ça faisait de drôles de bruits : Tac! Tac! Tac ! Tac !» [21] 